\title{
A Two-Picture Acquisition System for 3D Geometry and Light Reflection Modeling of Faces for Cosmetic Applications
}

\author{
Hussam YOUSEF, Céline LOSCOS*, Régis HUEZ, Michel HERBIN \\ University of Reims Champagne-Ardenne, CReSTIC-SIC EA 3804, Reims, France
}

http://dx.doi.org/10.15221/13.031

\begin{abstract}
Objectifying the visual effects of cosmetic products is a major concern for both manufacturers and consumers. One of the most complex effects of cosmetic products is the transparent aspect of the top layers of the skin, called translucency. Most evaluation methods of this phenomenon are still very subjective due to the complex visual quantification. For evaluating the effects of cosmetic products on skin translucency, we have developed an acquisition system to recover facial geometry and translucency coefficients. The equipment is designed to be low-cost and portable, and our reconstruction procedure needs only two photographs. The algorithm for 3D reconstruction uses one photograph of the face of a human subject on which was projected colored structured light to extract a 3D surface. An image-based approach deduces an RGB translucency value for a skin patch from a unique non-calibrated photograph. The proposed system was proven robust by means of experimentations conducted on 20 subjects and three different cosmetic products. We show that an image-based evaluation procedure is sufficient to consistently differentiate translucent effects of a range of cosmetics on a same skin, even from a unique non-calibrated image. The extracted parameters can be used for assessment of the effect of a product or for simulating this effect on a face.
\end{abstract}

Keywords: 3D face acquisition system, structured light scanning, BSSRDF, portable devices

\section{Introduction}

Facial capture is a well addressed topic with applications in many industries with high impact such as gaming and movies. It relies on the acquisition of face geometry and material properties of the face, i.e., how each part of the face reacts to light. In this paper, our target application is cosmetics, in particular the modification of the skin appearance relatively to the illumination when applying cosmetic products. We present a new acquisition system that is able to reconstruct facial geometry as well as to recover some of the material coefficients.

Different means can be used for facial reconstruction, for example, structured-light scanning, photogrammetry reconstruction from different photographs, or laser scanning. Some systems rely on the person remaining static which is very hard for humans always subject to small, uncontrollable movements. Other systems need synchronized capture for several takes made at once. The different material properties of the face add to the complexity of the process. Facial reconstruction is often made difficult due to human movement between takes. The system we propose needs only one shot of a face on which colored structured light is projected.

Light-skin interaction modeling involves complex material such as for eye balls, skin, and hair. In this paper we concentrate on the human skin, and in particular, on the translucency aspect, which is the effect due to the light entering the first layer of the skin, producing light diffusion below the surface. Translucency is estimated via an image-based approach that only needs one photograph as input.

The paper is structured as follows. In section 2, we introduce the skin reflectance properties and review previous approaches on image-based skin reflectance reconstruction. We present an overview of the acquisition procedure and the equipment used in section 3. In section 4 , we explain our method to extract BSSRDF parameters from a unique RGB photograph. The evaluation procedure is and the results are given in section 5 .

\footnotetext{
*celine.loscos@univ-reims.fr
} 


\section{Previous work}

Skin reflectance properties have been studied in two different manners. One relies on its biological structure, building on the different skin layers to justify the relation between the incoming and the outgoing light. The other relies on the visual aspect, starting from images to validate a reflectance model. In fact, some methods mix both approaches to reach a reliable but manageable model in its computational cost. In this section, we review the different skin reflectance models proposed in the literature, and we discuss the different approaches proposed to extract parameters from photographs of human faces.

\subsection{Skin reflectance models}

Human skin is composed of several layers that interact one with another. The outside appearance (shape, color and texture) of human skin depends on these different layers and how they react to incoming light. The behavior of this complex material interacting with light varies over time depending on various conditions such as emotions or ambient temperature. This material was approximated by a subsurface scattering model [1] relating the incoming light in one surface point $x_{i}$ to an outgoing ray in another surface point $\mathrm{x}_{\mathrm{o}}$ (see Figure $1(\mathrm{a})$ ).

Actually, as illustrated in Figure 1(b), one can consider the set of all outgoing light rays that relate to one incoming light ray $\mathbf{w}_{\mathbf{i}}$ at point $\mathrm{x}_{\mathrm{i}}$. This incoming ray bounces in different manners. Part of it is reflected at the same point and comes out as $\mathbf{w}_{\mathrm{oi}}$, another part is scattered within the material and comes out at different points of the surface, such as $\mathbf{w}_{\mathbf{o} 1}$ in point $\mathrm{x}_{1}$ and $\mathbf{w}_{\mathrm{o} 2}$ in point $\mathrm{x}_{2}$, while another part is absorbed by the material. The light is therefore diffused and scattered within the matter before exiting the surface. The set of outgoing light rays related to one incoming light ray describe a diffusion profile.

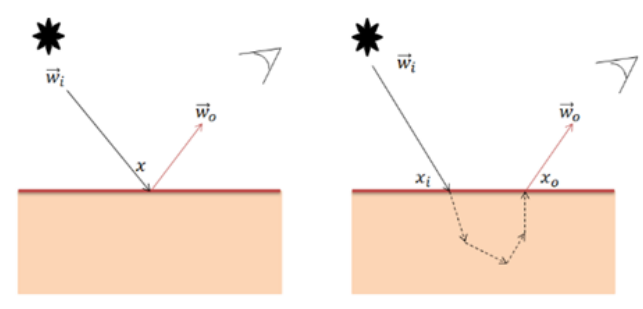

(a)

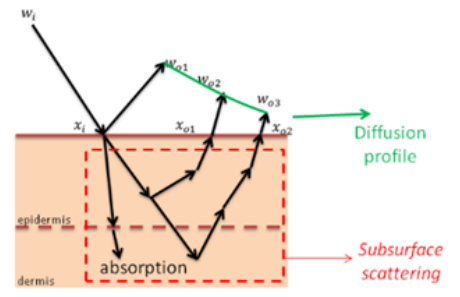

(b)

Figure 1. (a) (Left) a direct reflection of the incoming light ray (BRDF model). (Right) an incoming light ray entering the media for sub-surface scattering before exiting in a different point. (b) BSSRDF approximation of the set of outgoing light rays as a diffusion profile.

Several models were proposed to simulate the interaction of light with human skin. Adaptations of the BRDF model were proposed [2,3,4] but it was demonstrated [5] that a BRDF model is not sufficient to simulate the absorption and reflection of human skin. A Bidirectional Surface Scattering Reflectance Distribution Function (BSSRDF) approximates better light transport within the skin medium as described in Figure 1(b). Several BSSRDF models were proposed. Pharr and Hanrahan [6] use diffusion equations with a Monte Carlo integration to solve a radiant transfer equation. Krishnaswamy and Baranovski [7] propose a model based on biophysics of height dimensions. Both models are difficult to use because of their complexity and computational time. Diffusion theory was introduced for simulating multiple scattering effects by Stam [8]. It was revisited by Jensen et al. [9], extracting an exact term for simple diffusion out of complex geometry and using adequate sampling. Their resulting model computes diffuse reflection as a function of translucency parameters. This model is the most widely used, and has inspired several academic work $[10,11,12,13,14,15,16,17]$ and is a rendering asset in movie post-production software encouraged by the implementation suggestions of [18]. Another simulation model [19] was proposed to represent the multi-layer effect on skin translucency. However, measuring and setting parameters for each material layer is very difficult. By its simplicity and quality, the Jensen et al. [9]'s BSSRDF model was chosen as a base of our cosmetic evaluation procedure.

\subsection{Skin translucency acquisition methods}

Several methods were proposed to extract translucency from real data. Those which really quantify BSSRDF parameters use different sets up based on light ray(s) to study the light diffusion. Jensen et al. [9] use a white light source from which rays are gathered through two lenses to concentrate on one 
surface point. The visually observed diffusion helps defining values for the diffusion and absorption parameters. Goesels et al. [12] presented another measuring system for inhomogeneous objects. A camera records the HDR images of a scene scanned by three lasers producing red, green, and blue light. The parameter extraction uses Jensen et al. [9]'s BSSRDF model. However, the acquisition process takes too much time to be applicable to human subjects. Besides, these two methods require a clinical approbation procedure in order to be used on humans.

Skin translucency can also be acquired using a system combining optical fibers and spectrometer [200]. A fiber source produces light while additional sensor fibers acquire the reflected light. The system can locally extract the diffusion parameters, but several acquisitions would be necessary to compute an average value. Besides, the contact of the acquisition system with the skin could induce skin reactions modifying a cosmetic effect. This is therefore too invasive for cosmetic evaluation.

Other methods use structured light to project colors, shapes or points [211, 10,11]. BSSRDF is solved for height dimensions for heterogeneous objects [211], a per-pixel diffusion profile is provided [10] or the diffusion equation are solved through finite differences [11]. None of these approaches were appropriate for a practical evaluation of cosmetic skin translucency. Their sensibility to ambient illumination makes these procedures not practical enough.

Munoz et al. [222] proposed a BSSRDF estimation from a single image. Their approach differs from ours on the assumptions made. They need to have an estimate of the geometry and the illumination direction to extract the parameters to solve through a linear system minimization. It works for optically thick, homogeneous materials. Our approach is different because we focus on human skin. Therefore we can make assumptions on some parameters and we do not need to know the light direction and the geometry. However, this approach could also be considered.

\section{Acquisition procedure}

Our system is very simple (see Figure 2(a)), and is composed of a white background screen, two spot light sources (Lastolite RayR8 c5600), and a digital camera (Canon EOS 5D). The acquisition procedure is as follows. Light sources are placed at 90 degrees one to another with respect to the subject and let on for one minute to prevent illumination variation while they warm up. Their color temperature is $5200 \mathrm{~K}$. If possible, environmental lighting is controlled (or turned off) but this is not compulsory. The digital camera is placed so that its direction bisects the angle between the two light sources and is situated at 1 meter of the subject. It is calibrated to acquire the maximum range of values with the given illumination. The focus is made on the subject face. A polarized filter is applied to diminish specular reflection. An example of an acquired photograph is shown in Figure 2(b). For the BSSRDF estimation a patch is selected on the cheek. Another shot is taken with colored structured light projected on the face (see Figure 2 (c)). While the system and procedure are simple, it is preferable to keep the same room conditions if the study requires consistency and/or comparison between different acquisitions made at different times.

The 3D geometric reconstruction follows the approach described in [23]. The choice and order of the colors is made so that there cannot be confusion about the line index. An image processing algorithm is applied to join colored lined together. This forms a set of curved lined. This curvature is used to extract depth information, and therefore to build a 3D surface. The algorithm used to extract translucency coefficients is explained in the next section.

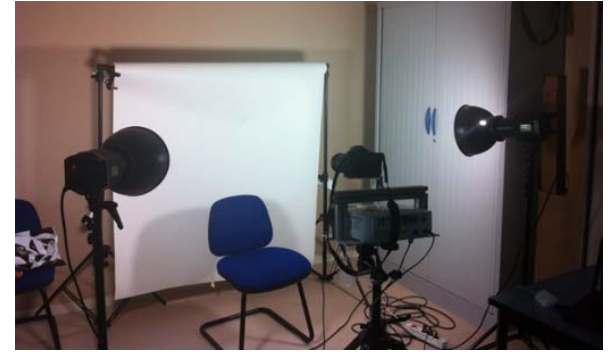

(a)

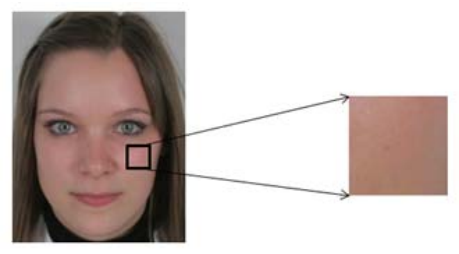

(b)

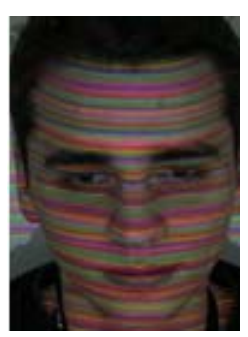

(c)

Figure 2 - (a) Our low-cost, portable acquisition system composed of a projector, two spot lights, a white screen, and a still camera. (b) A patch selected manually on the face cheek. (c) Projected colored structured light. 


\section{Skin parameter acquisition and reconstruction}

We estimate skin translucency parameters from a skin patch extracted from a unique image taken using the system described in section 3. Our approach builds on the BSSRDF implementation of Hery [18] and on the diffuse path approximation of Jensen and Buhler [24]. Skin translucency relates to a diffusion profile estimated from two coefficients: the scattering coefficient $\sigma_{s}{ }^{\prime}$ and the absorption coefficient $\sigma_{\mathrm{a}}$. We describe here how to estimate these coefficients from a human skin photograph, as a function of the reduced albedo $\alpha^{\prime}$ and the mean-free path $I_{d}$.

\subsection{Parameter definitions and equations}

Jensen and Buhler [24] proposed a new parameterization of the Jensen et al. [9]'s BSSRDF model so that the absorption and diffusion coefficients could be computed from the total skin diffusion, therefore directly from the skin color. From Stam [8] it can be assumed that simple diffusion is negligible for human skin. The total diffuse reflectance can therefore be approximated through four parameters, Rd $\left(x_{i}, \mathbf{w}_{i}, x_{0}, w_{o}\right)$, and only depends on the light entering and exiting points, $x_{i}$ and $x_{0}$, and incident and reflecting angles, $\mathbf{w}_{\mathbf{i}}$ and $\mathbf{w}_{\mathbf{l}}$. If assuming a uniform lighting, the BSSRDF can be approximated by a BRDF (same entry and exiting point) while modeling subsurface scattering.

The diffuse reflectance $R_{d}$ is defined as a function of function (three times), these one are no-linear. Firstly, the diffuse reflectance $R_{d}$ is a function of the reduce albedo a' and a factor $A$. Secondly, the factor $A$ is a function of $F_{d r}, F_{d r}$ being the mean diffuse reflectance of Fresnel. Thirdly $F_{d r}$ is a function of the relative refraction coefficient $\eta$. This last coefficient being obtains by the using of tables.

$R_{d}=f\left(A, \alpha^{\prime}\right)$

$A=f\left(F_{d r}\right)$

$F_{d r}=f(\eta)$

We use the relative refraction coefficient $\eta=1.3$ for human skin [25], $A$ is known and $R_{d}$ only depends on $\alpha^{\prime}$. Since there is no simple analytical solution to this equation, Hery [18] proposes the use of a look-up table created only once to compute a diffuse reflectance associated to $\alpha$ '.

The reduced albedo is connected to reduced scattering coefficient $\sigma_{s}$ ' and absorption coefficient $\sigma_{a}$ by another no linear function.

$\alpha^{\prime}=f\left(\sigma_{s}{ }^{\prime}, \sigma_{a}\right)$

In order to solve this equation, Jensen and Buhler [24] introduce the mean-free path $l_{d} \approx \frac{1}{\sigma_{t r}}$.

Thus if $I_{d}$ is known, $\sigma_{t r}$ can be computed and the reduced total extinction coefficient $\sigma_{t}^{\prime}=\sigma_{s}^{\prime}+\sigma_{a}$ calculated using an equation connecting $\sigma_{t}^{\prime}$ to $I_{d}$ and $\alpha^{\prime}$ :

$$
\sigma_{t}^{\prime}=f\left(\alpha^{\prime}, \sigma_{t r}\right)
$$

The translucency parameters to be computed are:

$\sigma_{s}^{\prime}=\alpha^{\prime} \sigma_{t}^{\prime}$ and $\sigma_{a}=\sigma_{t}^{\prime}-\sigma_{s}^{\prime}$

From equations (3) and (4) we can deduce that the evaluation of the translucency parameters only depends on $I_{d}$ and $\alpha^{\prime}$. We set $I_{d}=(1.8155,1.0213,0.6453)$ by Weyrich et al. [13] for each R,G,B channel and $\alpha^{\prime}$ is computed from $R_{d}$.

\subsection{Translucency parameters estimation}

Our procedure to compute the translucency parameters is the following. We use the system presented in section 3, respecting two constraints. First, the illumination needs to be locally ambient and uniform. It is ensured by the two spot lights directly illuminating the subject face, thus potentially overriding any other scene lighting effect. Second, the material can be considered as infinite [9]. This is approximated using a selected patch on the cheek (see Figure 2 (b)) for computations. 
As mentioned before, in our algorithm, only the multiple diffuse scattering is taken into account [8]. Our algorithm is as follows:

- the total diffuse reflectance $R_{d}$ is computed in RGB as the average of the skin color on the patch;

- from $R_{d}$, the albedo $\alpha^{\prime}$ is computed using a look-up table [18];

- the translucency parameters $\sigma_{s}{ }^{\prime}$ and $\sigma_{a}$ are then deduced from equations (3) and (4), using $I_{d}$ values given in section 4.1. and $\alpha^{\prime}$ previously computed.

\section{Results}

We conducted an experiment following the procedures described in section 5.1. From the collected data, we estimated $\sigma_{\mathrm{s}}{ }^{\prime}$ and $\sigma_{\mathrm{a}}$. Results are discussed in section 5.2. From this estimated coefficients, we demonstrate a statistical classification of the effect on skin translucency from the cosmetic products tested in this experiment. The statistical tests and their results are shown in section 5.3. Rendering results are shown in section 5.4 .

\subsection{Experiment procedure}

We ran an experiment to verify our assumption using our evaluation system. The experiment took place in a cosmetic company where our system was set up. The lighting in the room was kept constant during the day by closing the window curtains while maintaining on the main light sources of the room. We enrolled 20 volunteers, 10 women and 10 men, all Caucasian and healthy, who were properly informed of the experiment and who signed a consent form. The cosmetic products were chosen by the cosmetic company and were applied by one of the cosmetic employees, the same person for the full day of the experiment. Before and in-between takes, the subject face was cleansed on both sides. The scene is fixed, only the volunteers change [266]. The cosmetic products are each from a different brand, and we will name them A (hydrating), B (day cream), and C (BB cream) in this paper. We expect products $A$ and $B$ to have a very small effect on skin translucency while product $C$ to be significantly different since it is more covering with a more opaque-looking effect.

Five photographs per volunteer were taken. The first photograph is used for $3 \mathrm{D}$ reconstruction; the others are named generically $\operatorname{ImX}, X$ varying with the different skin conditions: the image $\operatorname{ImN}$ is taken with no cosmetic application, the images $\operatorname{ImA}, \operatorname{ImB}$ and $\operatorname{ImC}$ are acquired with the application of respectively the products $A, B$ and $C$ on the left hemiface of each volunteer. An example of these four photographs is shown in Figure 3 . With 20 subjects, this leads us to 80 colored photographs. No cosmetic product was applied on the right hemiface of the volunteers. A latency of ten minutes is observed before taking a picture to let the cream penetrate the skin. Once the photograph is taken the full face is cleansed. A delay of approximately 30 minutes is observed before applying the next product to let the skin rest and be free of irritations. The right hemiface is left with no makeup during the entire procedure to serve as a reference when comparing the product effects.

Two equal-sized patches are selected on the right and left cheeks of the face (see Figure 4) for all photographs, at the same position. This latter condition is observed as much as possible since the persons sit on the same seat but their position may vary slightly from one photograph to another. The patches are called ImXr and ImXI when they are extracted from image ImX. We thus have 8 patches per volunteer, or 160 patches in total.

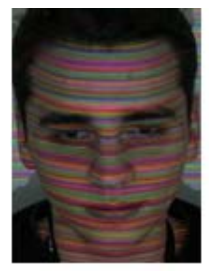

$\mathrm{L}$

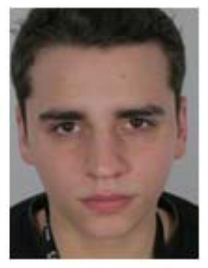

$\mathrm{N}$

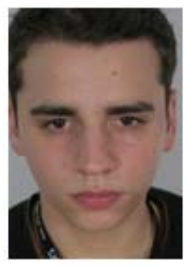

A

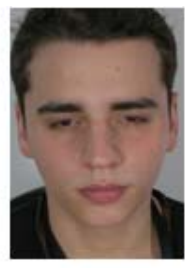

B

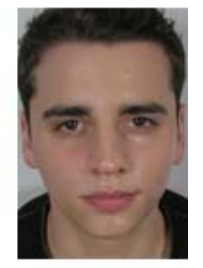

C

Figure 3. Five acquired photographs. L: with structured light. N: Neutral, no product was applied. A: with product A applied. B: with product $B$ applied. C: with product $C$ applied. 


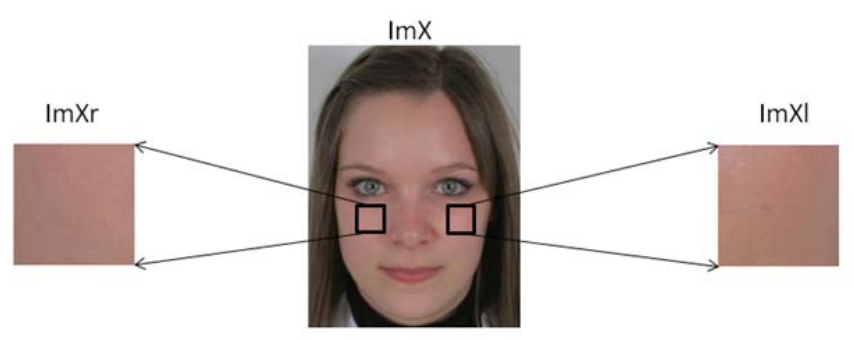

Figure 4. Image $\operatorname{ImX}$ and extraction of two patches: ImXr on right cheek (without cosmetic treatment) and ImXI on the left cheek (with cosmetic treatment).

\subsection{Estimation of the reduced scattering and absorption parameters}

Following the algorithm presented in section 4 , we extract 8 pairs (4 by hemiface) of translucency parameters, with RGB values from four photographs of a same person taken with our acquisition system. For each subject, we thus extract 48 values. An example of extracted parameters for subject 1 (see Figure 3) is shown in Table 1. In this table small variations can be noticed on the right hemiface while it is supposed not to have received any make up. Skin appearance may slightly change in between takes because it is a living material and may react to physiological (e.g., heat variation, irritation, etc.) or emotional changes. Besides, the position of the estimated patch may vary from one photograph to another. However, we demonstrate in section 5.3. that the differences between values are not statistically significant.

Table 1. Example of parameters $\sigma_{s}^{\prime}$ and $\sigma_{a}$ extracted from four photographs taken of the same subject (see Figure 3) under our experimental conditions. $\sigma_{s R}^{\prime}$ and $\sigma_{a R}$ are extracted on the right hemiface, with no cosmetic product applied on neither of the four photographs. $\sigma_{s L}^{\prime}$ and $\sigma_{a L}$ are extracted from the left hemiface where different cosmetic products were applied.

\begin{tabular}{|c|c|c|c|c|c|c|c|c|c|c|c|c|c|}
\hline & \multicolumn{3}{|c|}{$\sigma_{S^{\prime} R}^{\prime}$} & \multicolumn{3}{|c|}{$\sigma_{a R}$} & \multicolumn{3}{|c|}{$\sigma_{s L}^{\prime}$} & \multicolumn{4}{|c|}{$\sigma_{a L}$} \\
\hline & $\mathrm{R}$ & G & B & $\mathrm{R}$ & G & $B$ & $\mathrm{R}$ & G & B & & $\mathrm{R}$ & G & B \\
\hline $\operatorname{ImN}$ & 3.201 & 3.0392 & 4.0625 & 0.031 & 0.1017 & 0.1883 & 3.091 & 2.9846 & 3.958 & & 0.032 & 0.103 & 0.1928 \\
\hline $\operatorname{ImA}$ & 3.023 & 349 & 059 & 0.03 & 0 & 952 & 3.33 & 3.1026 & 4 & & 0.0 & 0.099 & 1734 \\
\hline ImB & 2.979 & 721 & 8121 & 0.033 & 1 & 1995 & 3.37 & .20 & 4 & & 0.0 & 0 & 1731 \\
\hline $\operatorname{ImC}$ & 3.213 & 3.0336 & 4.1740 & 0.031 & 0.1019 & 0.1837 & 3.356 & 3.4351 & 4.473 & & 0.029 & 0.090 & 0.1723 \\
\hline
\end{tabular}

The extracted values do not have a proven physical meaning as such. We did not compare them with other measuring method. What interests us in this paper is the significant difference of translucency between two patches rather than the physical meaning of the extracted parameters. However, these values could be used for simulating skin BSSRDF in computer graphics applications. The changing behavior of these estimated data, statistically studied in the next section, could be used for translucency variation effects when rendering a virtual face. An example of rendering using these coefficients on a neutral face is shown in Figure 5.

\subsection{Evaluation of the variation of cosmetic translucency}

The analysis of the clinical experiment is made on a small sample size (20). The translucency parameters are biological data and therefore depend on the skin of the volunteers. A statistical analysis is required because the biological variations can be important from one person to another. Besides, the translucency parameters can vary with the patch location on the volunteer face, with the lighting condition of the study and with the cosmetic products chosen. From the translucency data (parameters $\sigma_{s}{ }^{\prime}$ and $\sigma_{a}$ ) estimated in RGB from the 80 photographs (see section 5.1. ), we compute the statistical significance of differences between pairs of patches, using the Wilcoxon ranking test [27]. 


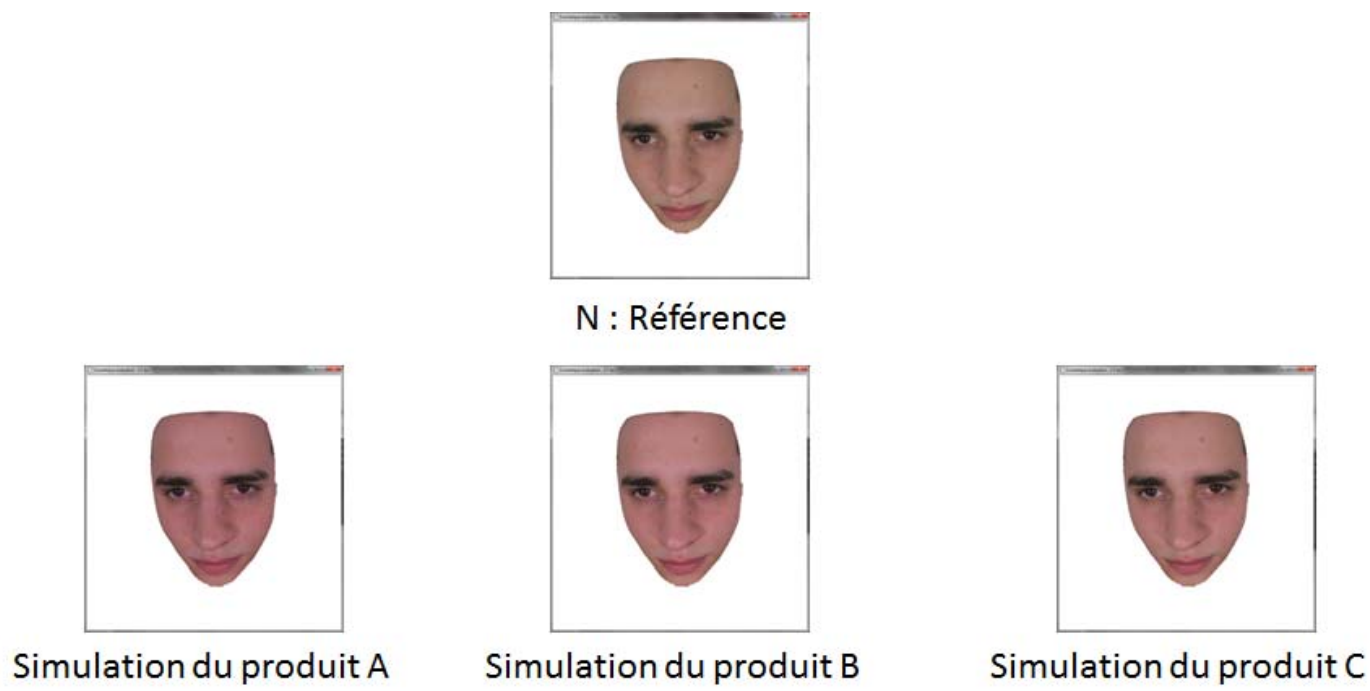

Figure 5 - BSSRDF parameters applied on image N (top), for simulating the effect of product $A$ (Bottom Left), B (Bottom Middle) and C (Bottom Right).

We analyzed the variations when using a cosmetic treatment on the left hemiface. The effect of one product $(A, B$ or $C)$ is controlled with no treatment reference $(N)((a),(b),(c))$. When comparing the 20 differences between $I \mathrm{mNI}$ and $I \mathrm{mAl}$, between $I \mathrm{mNI}$ and $I \mathrm{mBI}$, and between $I \mathrm{mNI}$ and $I \mathrm{mCl}$, we always obtain statistically significant differences (Wilcoxon tests, sample size $=20$ ). Even, their differences are highly significant $(p<0.01)$ for the R component when comparing $\operatorname{ImNI}$ with $\operatorname{ImAl}$ and $\operatorname{ImBI}$, and the $G$ component when comparing $I \mathrm{mNI}$ with $\mathrm{ImCl}$. Thus, all products $\mathrm{A}, \mathrm{B}$ and $\mathrm{C}$ have an effect on translucency when compared to no product application.

However, we could not discriminate the translucency effects of products $A$ and $B$. The differences between $\mathrm{ImBI}$ and $\mathrm{ImCl}$ are statistically significant for the green component of our translucency parameters $\sigma_{\mathrm{s}}{ }^{\prime}$ and $\sigma_{\mathrm{a}}$. Product $\mathrm{C}$ statistically increases the reflection and decreases the absorption in the green bandwidth when comparing to $\mathrm{B}$. As we could not statistically differentiate the translucency impact of $C$ over $A$, we can conclude that the effect of product $C$ on skin translucency is higher than the one of $\mathrm{A}$ or $\mathrm{B}$. This result is expected because $\mathrm{C}$ is more skin covering than $\mathrm{A}$ or $\mathrm{B}$.

\subsection{Discussion}

The obtained results of this study correspond to our intuitions on the translucency effect of the applied products. We demonstrated that the application of the tested cosmetic products decreases skin translucency. It is interesting to see that products A and B are difficult to differentiate. Our methodology is able to validate the system set up, thus the quantification results. In particular, we measured the impact of the left-right effect as well as the time consistency.

\section{Conclusions}

This paper presents a novel, portable and low-cost methodology for the cosmetic evaluation of human skin translucency, with a robust estimation of the parameters from a unique photograph. Experiments were conducted on 20 Caucasian participants that statistically verify the validity of the approach. We demonstrated through statistical measurements the ability of a model based on the computer graphics BSSRDF to quantify cosmetic translucency changes. The benefits to cosmetic industries are twofold. First, we showed that a computer graphics model can be used to represent cosmetic translucency. Second, we demonstrated that a non-invasive, image-based approach, is robust enough to conclude on visual cosmetic translucency effects.

Our method can estimate translucency parameters from a unique photograph. The parameters satisfied the statistics measured in this paper. Future work could be done to physically validate them so that they could be used for simulation. 


\section{References}

1. Nicodemus F., Richmond J., Hsia J., Ginsberg I., Limperis T., (1977): "Geometric considerations and nomenclature for reflectance." Monograph 161, National Bureau of Standards (US).

2. Marschner S. R., Westin S. H., Lafortune E. P. F., Torrance K. E., Greenberg D. P. (1999): "Image-based BRDF measurement including human skin." In Proceedings of 10th EUROGRAPHICS Workshop on Rendering, pp 139-152.

3. Hanrahan P., Krueger W., (1993): "Reflection from layered surfaces due to subsurface scattering." In Proceedings of the 20th annual conference on Computer graphics and interactive technique ACM SIGGRAPH, pp 165-174.

4. Stam J., (2001): "An illumination model for a skin layer bounded by rough surfaces." In Proceedings of the12th EUROGRAPHICS Workshop on Rendering, pp 39-52.

5. Murakami H., Horii T., Tsumura N., Miyake Y., (2002): Measurement and simulation of 3d gonio spectral reflectance of skin surface. Digital biocolor journal Vol. 93, pp 21-26.

6. Pharr M., Hanrahan P., (2000): Monte Carlo evaluation of non-linear scattering equations for subsurface reflection. ACM SIGGRAPH '00: Proceedings of the 27th annual conference on Computer graphics and interactive techniques, pp 75-84.

7. Krishnaswamy A., Baranoski G., (2004): "A biophysically-based spectral model of light interaction with human skin." Computer Graphics Forum, Vol. 23 No. 3, pp 331-340.

8. Stam J., (1995): Multiple scattering as a diffusion process. In EUROGRAPHICS Rendering Workshop.

9. Jensen H., Marschner S., Levoy M., Hanrahan P., (2001): A practical model for subsurface light transport. In ACM SIGGRAPH '01: Proceedings of the 28th annual conference on Computer graphics and interactive techniques, pp 511-518.

10. Tariq S., Gardner A., Llamas I., Jones A., Debevec P., Turk G.,(2006): "Efficient estimation of spatially varying subsurface scattering parameters." In Vision, Modeling, and Visualization, pp 165-174.

11. Mukaigawa Y., Suzuki K., Yagi Y., (2008): "Analysis of subsurface scattering under generic illumination." Proc. IEEE ICPR, 19th International Conference on Pattern Recognition, pp 1-5.

12. Goesels M., Lensch H., Lang J., Fuchs C., Seidel H., (2004): "DISCO: acquisition of translucent objects." ACM SIGGRAPH, In ACM Transactions on Graphics (TOG) Vol. 23, No. 3, pp 835-844.

13. Weyrich T., Matusik W., Pfister H., Bickel B., Donner C., Tu C., Mcandless J., Lee J., Ngan A., Jensen H., Gross M., (2006): "Analysis of human faces using a measurement-based skin reflectance model." ACM SIGGRAPH, In ACM Transactions on Graphics (TOG) Vol. 25, No. 3, pp 1013-1024.

14. Tsumura N., Ojima N., Sato K., Shiraishi M., Shimizu H., Nabeshima H., Akazaki, Hori K. S., Miyake Y., (2003): "Image-based skin color and texture analysis/synthesis by extracting hemoglobin and melanin information in the skin." ACM Transactions on Graphics (TOG) Vol. 22 No. 3, pp770-779.

15. Mashita T., Mukaigawa Y., Yagi Y., (2011): "Measuring and modeling of multi-layered subsurface scattering for human skin." In $\mathrm{HCl} 13$, pp 335-344.

16. Donner C., Weyrich T., D'Eon E., Ramamoorthi R., Rusinkiewicz S., (2008): "A layered, heterogeneous reflectance model for acquiring and rendering human skin." In ACM Transactions on Graphics (TOG) Vol. 27, No. 5, p 140.

17. Dipanda A., Woo S., (2005): "Towards a real-time 3D shape reconstruction using a structured light system." Pattern Recognition 38, pp 1632-1650.

18. Hery C., (2003): "Implementing a skin bssrdf." RenderMan: Theory and Practice, ACM SIGGRAPH Course Notes, Course 9, pp 73-88.

19. Donner C., Jensen H., (2005): "Light diffusion in multilayered translucent materials." In ACM Transactions on Graphics (TOG) Vol. 24, No. 3, pp 1032-1039.

20. Nickell S., Hermann M., Essenpreis M., Farrell T., Krämer U., Patterson M., (2000): "Anisotropy of light propagation in human skin." Physics in medicine and biology, Vol. 45 No.10, pp 2873-2886.

21. Peers P., Berge K. V., Matusik W., Ramamoorthi R., Lawrence J., Rusinkiewicz S., Dutre P., (2006): "A compact factored representation of heterogeneous subsurface scattering." ACM SIGGRAPH. In ACM Transactions on Graphics (TOG) Vol. 25, No. 3, pp. 746-753.

22. Munoz A., Echevarria J. I., Seron F., Lopez Moreno J., Glencross M., Gutierrez D., (2011): "BSSRDF estimation from single images." Computer Graphics Forum Vol. 30 No. 2, pp 455-464.

23. Yousef H., Huez R., Hussenet L., Herbin M. (2011); "An innovative approach in structured light systems." Proc. SPIE 7864, Three-Dimensional Imaging, Interaction, and Measurement, 78640N (January 27). 
24. Jensen H., Buhler J., (2002): "A rapid hierarchical rendering technique for translucent materials." SIGGRAPH '02: Proceedings of the 29th annual conference on Computer graphics and interactive techniques, pp 576-581.

25. Tuchin V., (2000): "Light Scattering Methods and Instruments for Medical Diagnosis." SPIE press. Tissue Optics.

26. "Guidelines for the evaluation of the efficacy of cosmetic products"(May 2008). Colipa Guidelines, The European Cosmetics Association, http://www.colipa.eu/about-colipa.html.

27. Wilcoxon F., (1945): "Individual comparison by ranking methods." Biometrics Vol. 1, pp 80-88. 